\title{
From Magnetite Nanoparticles to Thin Film and Nano-lithographed Arrays
}

Geronimo Perez $^{1}$, Elisa Saitovitch ${ }^{2}$ and Guillermo Solorzano ${ }^{3}$

${ }^{1}$ National Institute of Metrology, Rio de Janeiro, Rio de Janeiro, Brazil, ${ }^{2}$ Brazilian Center for Research in Physics, Rio de Janeiro, Rio de Janeiro, Brazil, ${ }^{3}$ Pontifical Catholic University of Rio de Janeiro, Rio de Janeiro, Rio de Janeiro, Brazil

Magnetite sputtering targets were produced by compression of magnetite nanoparticles. These nanoparticles were obtained by co-precipitation from the iron salts $\mathrm{FeSO}_{4}$ and $\mathrm{FeCl}_{3}$ in the ratio $\mathrm{Fe}(\mathrm{II}): \mathrm{Fe}(\mathrm{III})=1: 2$ with $\mathrm{NH}_{4} \mathrm{OH}$ solution addition. Structural characterization of the samples was conducted using a Siemens D5000 X-Ray diffractometer and a field emission JEOL 2100F TEM operating at $200 \mathrm{kV}$ and. The magnetite nanoparticles show a homogenous size distribution of spheroidal nanoparticles as observed in the bright field micrograph of Fig.1a and in the detail of Fig.1b, and the respective indexed SAED pattern of the Fig.1c exhibits the typical rings of polycrystalline magnetite structure. The magnetic behavior was observed by vibrating sample magnetometer (VSM) and by Mossbaïer spectroscopy. Then, $12 \mathrm{~g}$ of these magnetite nanoparticles were compressed to 7 Tons with a hydraulic press into a circular metal container 2.5 inches in diameter and $2 \mathrm{~mm}$ deep, to obtain the magnetite target for sputtering. These targets were used in the production of several magnetite thin films using the sputtering model ATC-2400 AJA International in RF (radio frequency source) mode on silicon substrates. The formation of the magnetite after the deposition was confirmed by structural and magnetic characterization: The structures of the samples were characterized by $\mathrm{X}$ ray diffraction (XRD). The patterns were recorded on a PANalyticalX'Pert PRO X-Ray diffractometer using $\mathrm{Cu}-\mathrm{K}_{\alpha}$ radiation $(\lambda=1.5418 \AA)$ and equipped with a $\mathrm{X}^{\prime}$ Celerator detector of 128 channels. The diffraction patterns were obtained by increasing $2 \mathrm{q}$ from $20^{\circ}$ to $70^{\circ}$ with step of $0.02^{\circ}$ and magnetic properties were measured using a Quantum Design Versalab 3 Tesla Cryogen-free vibrating sample magnetometer (VSM), at longitudinal and transverse (perpendicular to the magnetic field) directions. Some samples showed a magnetic saturation near $85 \mathrm{emu} / \mathrm{cm}^{3}$ at longitudinal direction (easy magnetization direction). Once the best sputtering conditions were found, the magnetite periodic arrays of basic shapes were produced by electron beam lithography followed by lift-off process and sputtering: a $1 \mu \mathrm{m}$ squares array and arrays of circles of $1 \mu \mathrm{m}, 500 \mathrm{~nm}$ and $250 \mathrm{~nm}$ in diameter formed of magnetite film $80 \mathrm{~nm}$ thick deposited by RF sputtering. Scanning electron microscopy (SEM) and atomic force microscopy (AFM) images provide additional morphological and topographical information. The AFM provides good topography and thickness information. Fig.2a shows AFM topography image and its respective dimensional profile of the circle pattern, Fig.2b exhibit the SEM image of the same sample. Mechanical test showed that the deposited magnetite thin films exhibit good adhesion and abrasion resistance. 


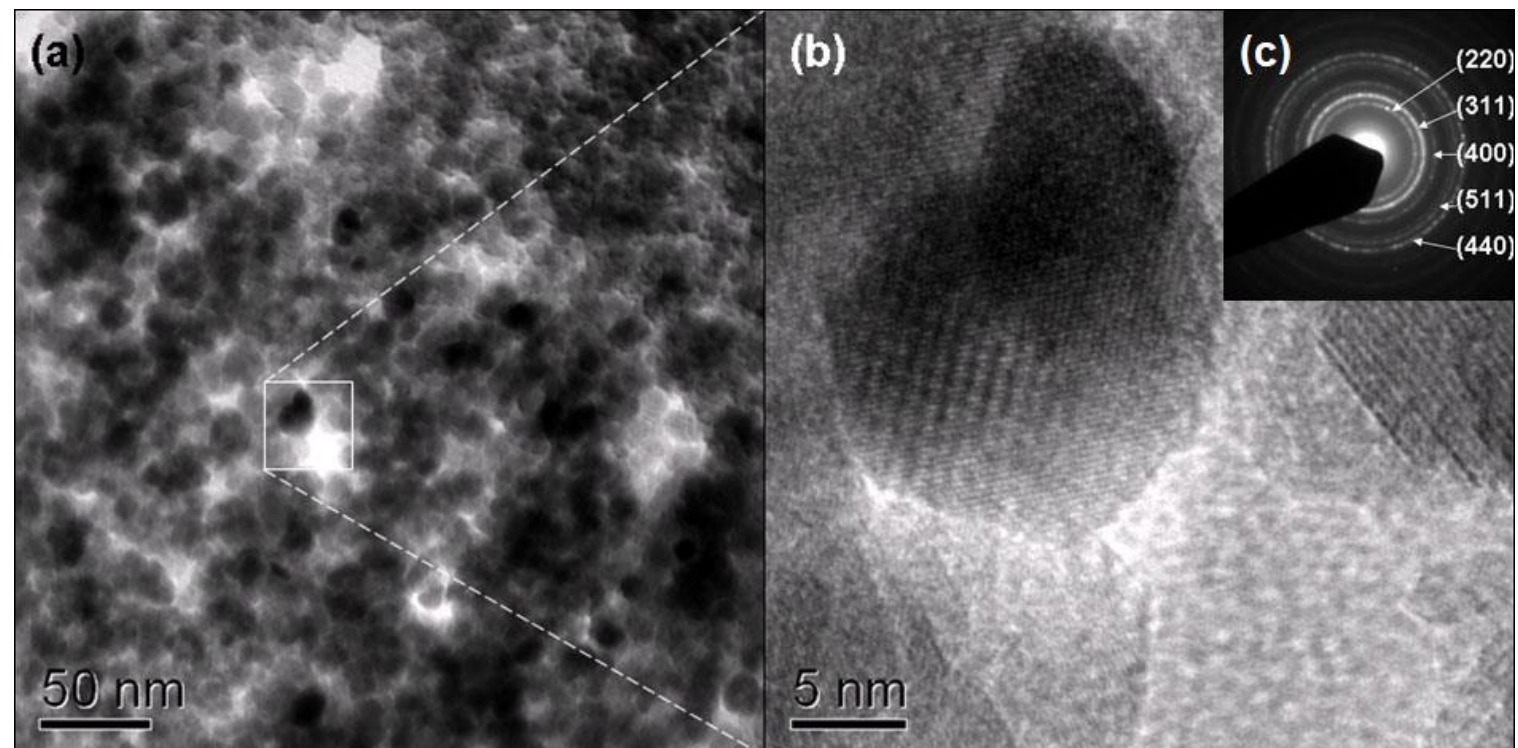

Figure 1. TEM micrographs of the magnetite nanoparticles: (a) Bright field, (b) multibeam, (c) selected area diffraction pattern.
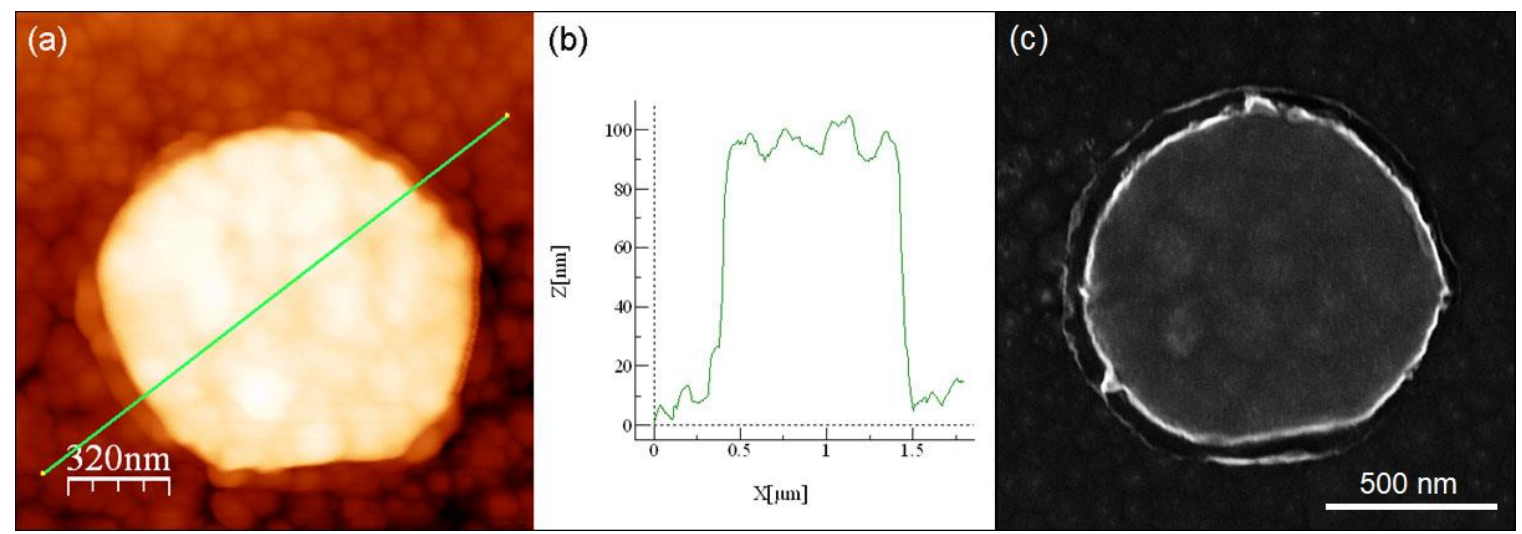

Figure 2. Images obtained of the circle pattern array: (a) AFM image of magnetite $1 \mu$ circle, (b) dimensional profile of respective AFM image, (c) SEM image of magnetite $1 \mu \mathrm{m}$ circle.

\section{References}

1. H. Takahashi, et al, J Appl Phys, 2003,93: 8029-8031.

2. X. L. Tang, et al, Journal of Solid State Chemistry 179 (2006) 1618-1622.

3. K. I. Aoshima and S. X. Wang, J. Appl. Phys. 93 (2003) 7954.

4. L. Pan, et al, Thin Solid Films 473 (2005) 63- 67. 